\title{
Optimal thickness of rectangular superconducting microtraps for cold atomic gases
}

\author{
A. Markowsky, ${ }^{1}$ A. Zare, ${ }^{1}$ V. Graber,${ }^{1}$ and T. Dahm ${ }^{1,2}$ \\ ${ }^{1}$ Institut für Theoretische Physik and Center for Collective Quantum Phenomena, \\ Universität Tübingen, Auf der Morgenstelle 14, D-72076 Tübingen, Germany \\ ${ }^{2}$ Universität Bielefeld, Fakultät für Physik, Postfach 100131, D-33501 Bielefeld, Germany
}

(Dated: May 27, 2022)

\begin{abstract}
We study superconducting microtraps with rectangular shapes for cold atomic gases. We present a general argument why microtraps open, if brought close to the surface of the superconductor. We show that for a given width of the strips there exists an optimal thickness under which the closest distance of the microtrap from the superconductor can be achieved. The distance can be significantly improved, if the edge enhancement of the supercurrent near edges and corners is exploited. We compare numerical calculations with results from conformal mapping and show that conformal mapping can often give useful approximate results.

PACS numbers: 37.10.Gh, 34.35.+a, 74.25.N-, 74.78.-w
\end{abstract}

Microstructured magnetic traps are useful devices for the storage and manipulation of ultracold atoms and Bose-Einstein-Condensates [1. In particular they allow studying the interaction of ultracold atoms with solidstate surfaces. However, the lifetime of the atomic clouds close to the surface is limited by noise radiation from current fluctuations in the conducting surfaces [2, 3]. Therefore, the use of superconducting materials for such devices is being investigated $4-10$. The energy gap of a superconductor strongly suppresses noise in the relevant frequency range and thus allows significantly longer lifetimes [11 14. Also, the use of superconductors promises the construction of hybrid quantum systems and coupling with superconducting electronic elements like Josephson Junctions, SQUIDs, or microwave cavities as a possible route for quantum computation [15, 16].

One drawback of superconducting microtraps is the strong redistribution of current due to the Meissner effect. Cano et al have shown that the Meissner effect in superconducting microtraps lowers the trap depth and decreases the trapping frequencies [7, 17] hampering the close approach of the atomic cloud to the superconductor surface. The closest distance reported so far in a superconducting microtrap has been $20 \mu \mathrm{m}$ [13. In principle, trap depth and trapping frequencies can always be increased with stronger currents and fields. However, the maximum current is limited by the critical current density in a superconducting material. As a possible way out of this difficulty it has been suggested to utilize the vortex state of type-II superconductors in which magnetic field partially penetrates the superconductor and reduces the Meissner effect [18, 19. However, this reintroduces noise radiation coming from the normal conducting cores of the vortices and mitigates the use of the superconducting material [20.

In this work, we study how the Meissner effect lowers the magnetic trap depth. The screening current due to the Meissner effect becomes particularly strong near corners and edges of a superconducting structure [21. Here, we show that this edge enhancement of the screening current in a microtrap formed by a microstrip of rectangular cross-section can be utilized to bring a microtrap significantly closer to the surface of the superconductor. This allows a stronger atom-surface coupling without the need to enter the vortex state. We perform calculations of the current and field distributions in the vicinity of the microstrip and show that there exists an optimal strip thickness at which the closest distance to the surface can be achieved.

In superconducting microtraps cold atoms with magnetic moments are caught in magnetic field minima. A magnetic field minimum can typically be created by the inhomogeneous field of a conducting wire and a homogeneous external magnetic field perpendicular to the wire 1. In such a situation there will always be a magnetic field zero in the plane perpendicular to the wire. There is a fundamental reason why magnetic microtraps open in the vicinity of the surface of a superconductor, which derives from Ampere's law and the Meissner effect: the radial trapping frequency is proportional to the normal derivative of the tangential magnetic field component $\frac{\partial B_{t}}{\partial n}$ near the surface. Due to Ampere's law we have $\frac{\partial B_{t}}{\partial n}=\frac{\partial B_{n}}{\partial t}$. The normal component $B_{n}$ is continuous at a surface. As $\vec{B}=0$ inside the superconductor, it follows $\frac{\partial B_{n}}{\partial t}=0$ at the surface. Therefore, the radial trapping frequency approaches zero when a superconducting surface is approached and the trap has to open.

There are two ways out of this difficulty, which will be studied in the following. First of all, the above argument is true only on lengthscales larger than the London penetration depth $\lambda$, because the magnetic field inside the superconductor decreases continuously to zero on that scale. Secondly, there can be high screening current flowing in the superconductor, which may create high local field gradients. This is particularly true near corners and edges. In the following we will study magnetic microtraps generated by a rectangular superconducting (thin film) strip and investigate the behavior near the corners.

The geometry is shown in the inset to Fig. 1 (a). The cross-section of the strip lies in the $x-y$-plane with a current $I$ flowing through in negative $z$-direction. A con- 
stant external magnetic field $\vec{B}_{\text {ext }}$ is applied within the $x$ - $y$-plane, such that a magnetic field zero is created somewhere in the $x$ - $y$-plane outside the strip. In addition, a small offset field $\vec{B}_{\mathrm{o}}$ of 1 Gauss is applied in $z$-direction in order to avoid Majorana spin-flip losses [1. The offset field turns the magnetic field zero into a magnetic field minimum which locally acts as a harmonic oscillator potential for the atoms. We calculate the current distribution in the strip using the numerical method by Brandt and Mikitik 22. In this method the electromagnetic kernel is discretized on a grid for the strip cross-section and inverted in order to obtain the current distribution inside the strip. For the grid we choose an inhomogeneous mesh which is more closely spaced near the corners of the strip [21, 23]. After determination of the current distribution, the magnetic field distribution is obtained from the Biot-Savart law.

For a given direction of $\vec{B}_{\text {ext }}$ we vary the total current $I$ and the magnitude $\left|\vec{B}_{\text {ext }}\right|$ in order to minimize the distance of the magnetic field minimum from the strip under the following two constraints: 1) the magnitude of the magnetic field at all points on the surface of the superconductor is required to be larger than the trap minimum by an amount of $10 k_{B} T / \mu_{B}$ in order to ensure that the atoms cannot escape from the trap. In the calculations below we choose $T=200 \mathrm{nK}$, a typical value that can be achieved in cold atom experiments. 2) The current density in the strip is required to stay below the critical current density $j_{c}$ at all points. The critical current density generally depends on the quality of the material. Here, we will take $j_{c}=10^{6} \mathrm{~A} / \mathrm{cm}^{2}$ which is a typical value for $\mathrm{Nb}$ thin films [24]. Using this optimization procedure we finally determine the closest distance of the trap minimum allowed under the two constraints.

In order to check our results and study the influence of the penetration depth $\lambda$ we compare our numerical calculations with the method of conformal mapping [25]. Conformal mapping allows calculation of the field distribution and the surface current distribution semianalytically for polygonally shaped strips. This method is strictly valid only for penetration depth $\lambda \rightarrow 0$. However, it gives useful results as long as the trap minimum is farther away from the superconductor than the lengthscale $\lambda$, as can be seen from the results below. We use the conformal map for a rectangular strip that has been given by Chen et al [26]. Within the conformal mapping method the current density diverges at the corners, which is a consequence of the $\lambda \rightarrow 0$ limit. For our calculations we need to regularize these divergencies. We do so by requiring that the surface current density stays below $\lambda j_{c}$ at a distance of order $\lambda$ from the corners as a replacement for the second constraint mentioned above.

In Fig. 1 we show results for a $w=20 \mu \mathrm{m}$ wide strip. The penetration depth was taken $\lambda=100 \mathrm{~nm}$, a value appropriate for $\mathrm{Nb}$ thin films. Fig. 1. (a) shows the closest reachable distance $d$ from the strip as a function of film thickness $h$ for an external magnetic field applied in $x$ -

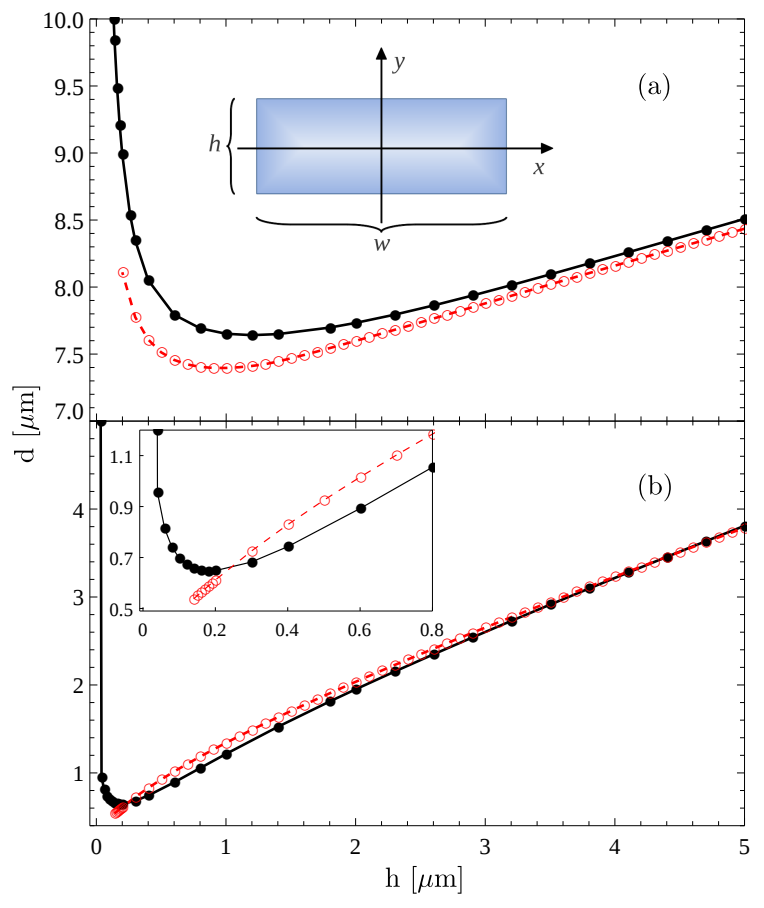

FIG. 1: (Color online) Closest reachable distance between the trap and a strip of width $w=20 \mu \mathrm{m}$ as a function of film thickness $h$ under the constaints discussed in the main text. Black solid circles show the results of the numerical calculation, red open circles are the results obtained from conformal mapping. a) The trap approaches the strip along the $y$-axis, external magnetic field is applied along the $x$-axis. Inset: Geometry of the strip. b) The trap approaches the strip along the $x$-axis, external magnetic field is applied along the $y$-axis. The inset shows an enlarged region near the minimum.

direction. In this case the magnetic field minimum always lies on the negative $y$-axis at the position $y_{0}$, i.e. $d=$ $\left|y_{0}\right|-h / 2$. The black solid circles show the results of the numerical calculation, while the red open circles are the results obtained from conformal mapping. Reducing the film thickness $h$ the reachable distance initially decreases slightly and reaches a minimum at a film thickness of $h=$ $1.2 \mu \mathrm{m}$ with a distance of $7.64 \mu \mathrm{m}$ from the strip. Further reduction of the film thickness leads to a quick increase of the closest distance. Both the numerical calculation and the conformal mapping method yield similar results. The conformal mapping method deviates from the numerical calculation on the order of the penetration depth and becomes invalid for smaller strip dimensions, as expected.

Qualitatively, the appearance of an optimum film thickness can be understood as follows: when the film thickness is large, the current flows mainly at the surface of the strip within a layer of thickness $\lambda$. For a fixed current density $j_{c}$ in the strip the total current $I$ then varies with the circumference of the strip. Decreasing $h$ leads to a slight decrease of $I \propto 1+h / w$. If one wants to keep the trap minimum at the same position, one has to decrease the magnitude of the external magnetic field by the same factor $1+h / w$. Then the magnetic field at 

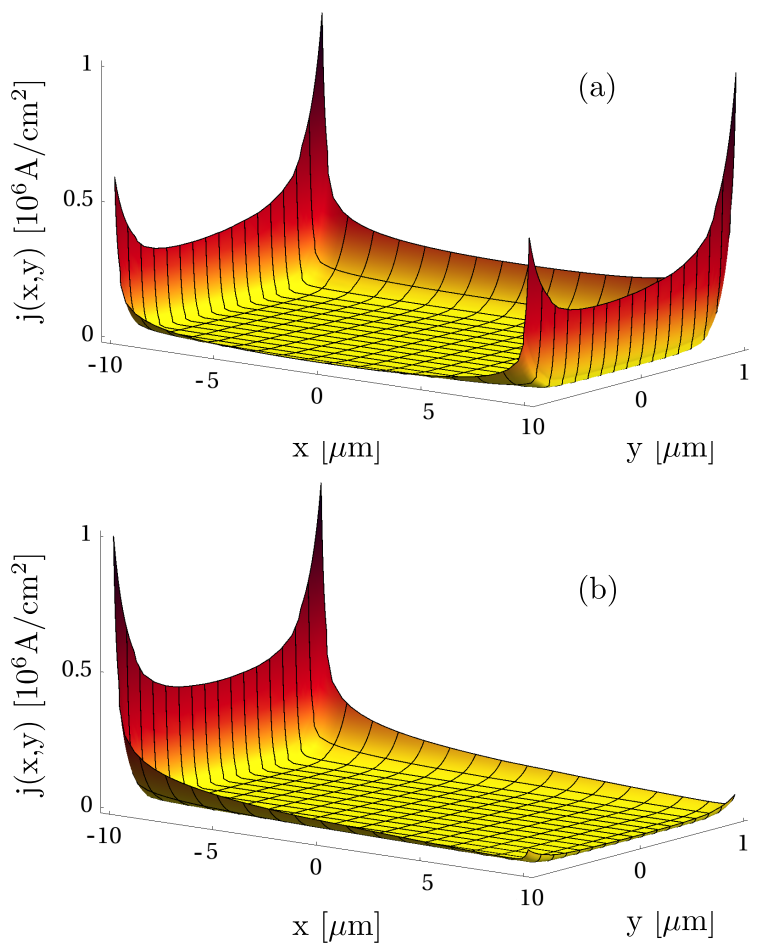

FIG. 2: (Color online) Current distribution at optimized distance inside a strip of width $w=20 \mu \mathrm{m}$ and thickness $h=2 \mu \mathrm{m}$. a) The external magnetic field is applied in $x$ direction and the trap minimum lies on the negative $y$-axis. b) The external magnetic field is applied in $y$-direction and the trap minimum lies on the positive $x$-axis.

the surface of the strip will increase because the external field has decreased while the field due to the current roughly remained constant because of the smaller thickness. In effect, the trap depth has increased and this means that the trap minimum can be brought closer to the strip. This explains the $\propto 1+h / w$ behavior of the minimum trap distance seen at larger values of $h$. When $h$ becomes smaller, the current flow in the strip becomes more homogeneous along the $y$-direction. Then the total current decreases more quickly $I \propto h$. In this case the trap depth decreases and the trap minimum has to be brought farther away from the strip in order to maintain the same trap depth.

The situation changes completely, if the external magnetic field is applied in $y$-direction and the trap minimum approaches the strip from its thin side along the $x$-axis, as shown in Fig. 1 (b). In this situation strong screening currents have to flow at the outer edges of the strip in order to screen out the external magnetic field from the inner part of the strip. Correspondingly, there exist much stronger field gradients in the vicinity of the trap minimum and the reachable distance initially decreases roughly proportional to $h$. This behavior stops when a film thickness of $h \approx \lambda$ is reached, because then the magnetic field can penetrate the strip and the screening is reduced. As Fig. 1 (b) shows, the optimum trap distance

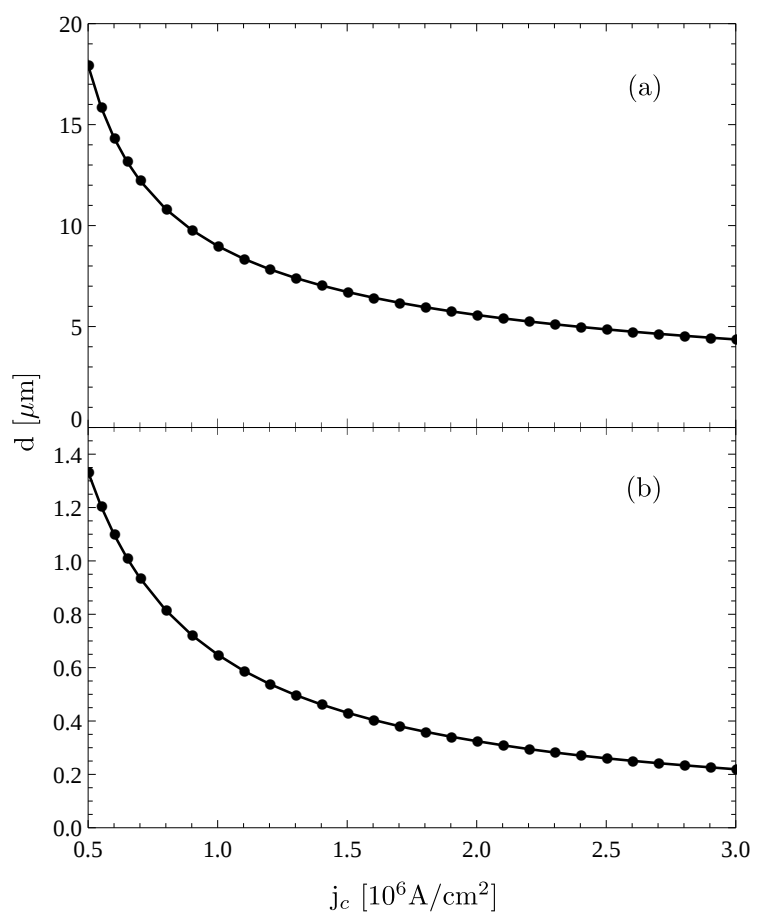

FIG. 3: Optimum distance as a function of the critical current density $j_{c}$ for a strip with width $w=20 \mu \mathrm{m}$ and thickness $h=0.2 \mu \mathrm{m}$. a) The external magnetic field is applied in $x$ direction and the trap minimum lies on the negative $y$-axis. b) The external magnetic field is applied in $y$-direction and the trap minimum lies on the positive $x$-axis.

is reached at a film thickness of $h=0.19 \mu \mathrm{m}$ with a distance of $0.65 \mu \mathrm{m}$ from the strip, about a factor of 12 times smaller than in Fig. 1 (a). The conformal mapping method does not show the increase of the trap distance at low values of $h$. This is due to the fact that in this range $h \sim \lambda$ the conformal mapping method is not valid anymore.

In Fig. 2 we show the current distribution inside a strip of width $w=20 \mu \mathrm{m}$ and thickness $h=2 \mu \mathrm{m}$ under optimized conditions. Fig. 2 (a) shows the current distribution when the trap minimum approaches the strip along the negative $y$-axis. In this case, the current flows mainly along the three edges of the strip away from the trap minimum. There is only small current flowing at the edge closest to the trap minimum. This is clear, because under optimized conditions the magnetic field becomes very small close to this edge and only small screening current has to flow here. The highest current density $\left(j_{c}\right)$ is found at the two corners opposite to the trap. Fig. 2 (b) shows the current distribution when the trap minimum approaches the strip along the $x$-axis. In this situation, the current flows mainly along the short edge of the strip opposite to the trap minimum. The current along the long edges of the strip can almost be neglected. This is due to the fact that the external magnetic field is applied along $y$-direction, which creates a strong screening current at the short outer edges of the strip 22]. The 

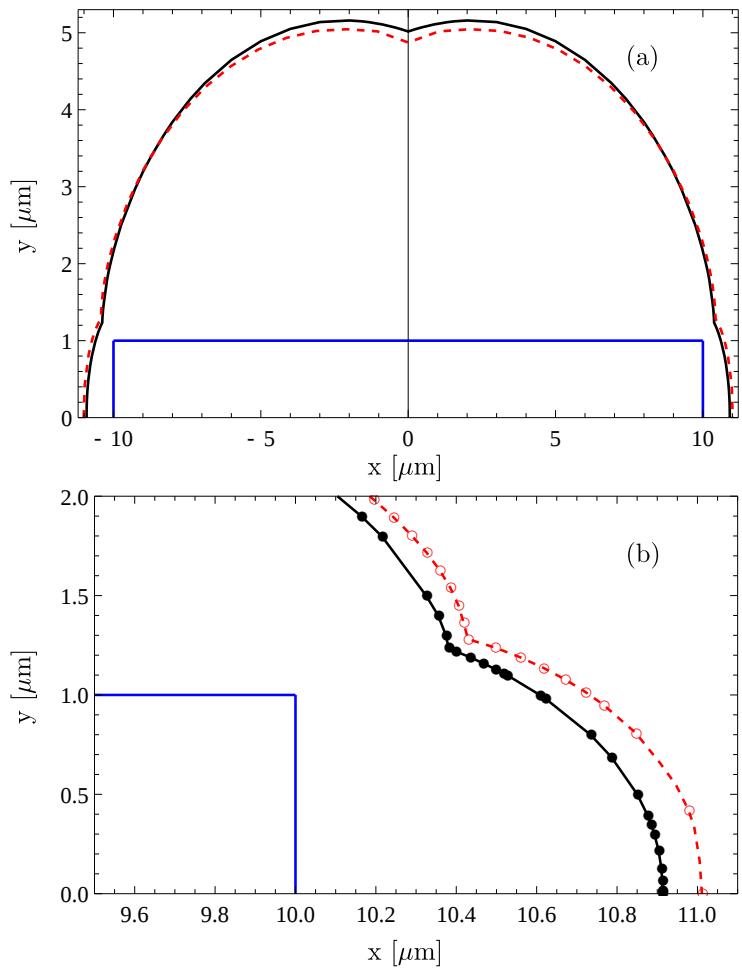

FIG. 4: (Color online) a) Locus of points of optimum distance for different directions of the external magnetic field. Here, a strip with width $w=20 \mu \mathrm{m}$, thickness $h=2 \mu \mathrm{m}$, and $j_{c}=3 \cdot 10^{6} \mathrm{~A} / \mathrm{cm}^{2}$ was taken. Black solid lines (solid circles) show the results of the numerical calculation, red dashed lines (open circles) are the results obtained from conformal mapping. b) shows an enlarged region near the corner of the strip.

highest current density is again found at the two corners opposite to the trap. The magnetic field gradient in the trap center is $4.5 \mathrm{~T} / \mathrm{m}$ for the case in Fig. 2 (a) and $9.9 \mathrm{~T} / \mathrm{m}$ for Fig. 2 (b).

The optimum distance that can be achieved under the two constraints above obviously depends on the quality of the material via the critical current density $j_{c}$. In Fig. 3 we demonstrate how the optimum distance varies with $j_{c}$ for a strip with width $w=20 \mu \mathrm{m}$ and thickness $h=0.2 \mu \mathrm{m}$ along the two coordinate axes. For $j_{c}=$ $3 \cdot 10^{6} \mathrm{~A} / \mathrm{cm}^{2}$, for example, one may approach the strip $4.5 \mu \mathrm{m}$ from the $y$-axis and $0.22 \mu \mathrm{m}$ from the $x$-axis.

In practice, an approach from the thin side of the strip may not always be feasible. Nevertheless, the edge enhancement of the screening current can still be exploited, if the trap is brought close to the corner of the strip. In Fig. 4 we show the locus of points of optimum distance for different directions of the external magnetic field for a strip with width $w=20 \mu \mathrm{m}$ and thickness $h=2 \mu \mathrm{m}$. Both the results from the numerical calculation and conformal mapping are shown. The results differ only by the order of the penetration length, as expected. As one can see, the optimum distance lies even closer to the strip in the vicinity of the corner. This is related to the stronger $l^{-1 / 3}$ divergence of the surface current in the corners in the limit $\lambda \rightarrow 0$, where $l$ is the distance from the corner 22].

To conclude, we have shown that for a rectangular superconducting strip of width $w$ there exists an optimal film thickness for which a microtrap can be brought closest to the surface of the superconductor. The closest approach can be achieved near the corners or short edges of the strip. This is due to the edge enhancement of the screening current. Near the corners distances in the submicrometer range can be realistically achieved. Such distances are essential for good control coupling in quantum computation [16, for coupling of atoms with a superconducting qubit via the near-field of a microwave cavity mode [15], or for studying the Casimir-Polder force.

We acknowledge useful discussions with D. Cano and J. Fortagh. This work was supported by the Deutsche Forschungsgemeinschaft via SFB/TR21.
[1] J. Fortagh and C. Zimmermann, Rev. Mod. Phys. 79, 235 (2007).

[2] M.P.A. Jones, C.J. Vale, D. Sahagun, B.V. Hall, and E.A. Hinds, Phys. Rev. Lett. 91, 080401 (2003).

[3] P.K. Rekdal, S. Scheel, P.L. Knight, and E.A. Hinds , Phys. Rev. A 70, 013811 (2004).

[4] T. Nirrengarten et al, Phys. Rev. Lett. 97, 200405 (2006).

[5] T. Mukai et al, Phys. Rev. Lett. 98, 260407 (2007).

[6] C. Roux et al, EPL 81, 56004 (2008).

[7] D. Cano et al, Phys. Rev. Lett. 101, 183006 (2008).

[8] V. Dikovsky et al, V. Sokolovsky, B. Zhang, C. Henkel, and R. Folman, Eur. Phys. J. D 51, 247 (2009).

[9] V. Sokolovsky, L. Prigozhin, and V. Dikovsky, Supercond. Sci. Techn. 23, 065003 (2010).

[10] D. Cano et al, Eur. Phys. J. D 63, 17 (2011).

[11] B.-S.K. Skagerstam, U. Hohenester, A. Eiguren, and P.K. Rekdal, Phys. Rev. Lett. 97, 070401 (2006).
[12] U. Hohenester, A. Eiguren, S. Scheel, E.A. Hinds, Phys. Rev. A 76, 033618 (2007).

[13] B. Kasch et al, New J. Phys. 12, 065024 (2010).

[14] R. Fermani et al, J. Phys. B 43, 095002 (2010).

[15] J. Verdu et al, Phys. Rev. Lett. 103, 043603 (2009); G. Bensky et al, Quantum Inf. Process. 10, 1037 (2011).

[16] R. Folman, Quantum Inf. Process. 10, 995 (2011); R. Salem et al, New J. Phys. 12, 023039 (2010).

[17] D. Cano et al, Phys. Rev. A 77, 063408 (2008).

[18] A. Emmert et al, Phys. Rev. A 80, 061604 (2009).

[19] M. Siercke et al, Phys. Rev. A 85, 041403(R) (2012); B. Zhang et al, Phys. Rev. A 85, 013404 (2012).

[20] G. Nogues et al, EPL 87, 13002 (2009).

[21] T. Dahm and D. J. Scalapino, J. Appl. Phys. 81, 2002 (1997).

[22] E. H. Brandt and G. P. Mikitik, Phys. Rev. Lett. 85, 4164 (2000). 
[23] E. H. Brandt, Phys. Rev. B 58, 6506 (1998).

[24] R. P. Huebener et al, J. Low Temp. Phys. 19, 247 (1975).

[25] P. Henrici, Applied and Computational Complex Analysis, Vol. I, Wiley (1988).
[26] D.-X. Chen, C. Prados, E. Pardo, A. Sanchez, and A. Hernando, J. Appl. Phys. 91, 5254 (2002). 